\title{
Diferencias de género en las prácticas e interacciones en los comentarios del diario online Emol.com
}

\author{
Gender differences in practices and interactions in news \\ commentaries in the digital newspaper Emol.com
}

\section{Karen Gheza Correa}

Universidad de Chile, Santiago, Chile.

karen.ghezaldgmail.com

\section{Resumen}

La investigación aborda las diferencias de género en las prácticas e interacciones en los espacios de participación del lector en la prensa online, específicamente, en los comentarios a las noticias del diario online Emol.com. De acuerdo a los objetivos propuestos y a la naturaleza del corpus de este estudio, se recurrió al análisis de contenido cómo técnica para describir y develar las diferencias de género, constatándose que, pese a la existencia de nuevas herramientas de participación en la prensa online que buscan enriquecer el debate público sobre asuntos sociales y políticos y asimismo, fomentar la incorporación de los ciudadanos/as en la esfera pública, estas herramientas más que aportar a la plena incorporación de las mujeres en el espacio público, refuerzan las desigualdades y los estereotipos de género.

Palabras claves: Diferencias de Género, Participación, Interacción, Prensa online.

\begin{abstract}
This study addresses gender differences in practices and interactions in participatory discussion forums for readers in online press, particularly in news commentaries in the digital newspaper www.Emol.com. According to the objectives and the nature of the corpus of this research, content analysis was conducted as a technique to describe and unveil gender differences, taking note that, despite the availability of new tools of participation in digital press, which are geared at enriching public discussion about social and political affairs and also fostering the incorporation of citizens into the public arena. These tools reinforce gender inequalities and stereotypes.
\end{abstract}

Keywords: Gender differences, Participation, Interactions, Online press. 


\section{Introducción}

Junto con el desarrollo de Internet y las Tecnologías de la Información, la prensa ha estado expuesta a las continuas transformaciones conforme van influyendo en ella los cambios socio-tecnológicos, siendo una de sus transformaciones más relevantes, la incorporación -en sus ediciones digitales- de espacios que promueven la participación e interacción de los lectores/as con los contenidos y entre sí. Estos "espacios de participación del lector" (Raimondo, 2012), agregan una nueva dimensión a los estudios en el campo de la prensa online que, con perspectiva de género, han dado cuenta del papel la mujer como profesional y como objeto y sujeto de la información. Aunque los estudios de prensa online que incluyen la variable de género tienen al menos una década de existencia, los estudios de prensa online y específicamente, aquellos que se hacen la pregunta sobre las diferencias de género en los comentarios a las noticias -ya sea a nivel de contenido o de participación- recién comienzan a articularse el año 2015, con el estudio de Emma Pierson la versión online de The New York Times, donde observó que sólo el $28 \%$ de los comentarios realizados en este medio fueron emitidos por mujeres (Pierson, 2015).

Ahora bien, por la preocupación de una sobrerepresentación masculina en los espacios de producción, y por el debate y difusión de discursos dentro de una sociedad democrática que debiese caminar hacia una representación equitativa de las opiniones de todos sus miembros, el presente trabajo tuvo como objetivo dar cuenta de las diferencias de género en las prácticas e interacciones de las lectoras y los lectores a partir de los niveles de participación -frecuencia de las opiniones emitidas por hombres y mujeres- en los comentarios del diario online Emol.com en las categorías noticiosas sobre educación, ciencia y tecnología, economía y política. La elección de estas temáticas se debió principalmente a que tratan asuntos propios del espacio público -espacio históricamente configurado por hombres- y con implicancias directas en el desarrollo del país y la calidad de vida de sus habitantes.

Asimismo, se establecieron cuatro objetivos específicos: (1) Determinar y establecer con qué frecuencia las lectoras y los lectores comentan las noticias en el diario online Emol.com; (2) Reconocer y determinar en cuáles de estas categorías de noticias nacionales (política, educación, economía y ciencias y tecnologíal las lectoras y los lectores del diario online Emol.com comentaron con mayor frecuencia; (3) Reconocer, comparar y determinar si los comentarios de las lectoras y los lectores en el diario Emol.com expresan distintos puntos de vista sobre un mismo asunto; y (4) Identificar, verificar y determinar la frecuencia y la naturaleza de los discursos sexistas presentes en los comentarios del diario online como respuesta a las opiniones de otros lectores/as.

\section{Antecedentes}

La prensa online, ante el riesgo de ser desplazada por las redes sociales donde los individuos son capaces de ejercer su libertad de expresión, comenzó a adaptarse a los nuevos desafíos comunicacionales de la era digital, incorporando en sus sitios herramientas que permitan (en mayor o menor medidal la participación e interacción de los ciudadanos con el medio, con el contenido y entre sí.

Dentro de estas nuevas herramientas de participación se encuentran los espacios de participación del lector, entendidos como aquellas instancias de un periódico online en donde se materializa la actividad del lector (Raimondo, 2012) y en donde estos "pueden manifestarse discursivamente produciendo enunciados, como por ejemplo sucede en las cartas de lectores, blogs (...), foros y los comentarios a las noticias" (Raimondo, 2014, p. 185).

Ahora bien, la prensa online $y$, específicamente, los espacios de participación del lector, al permitir la comunicación online de manera voluntaria, asincrónica y pública, se adscriben como objeto de estudio al campo de la Comunicación Mediada por Computadora (CMC). La CMC es probablemente una de las áreas más conocidas de Internet y una de las más utilizadas por sus usuarios a través de los sistemas de comunicación sincrónica, por un lado -chats, juegos de rol y mensajería instantáneos-, y los sistemas de comunicación asincrónica, por otro -correo electrónico, blogs y foros de discusión- (Gómez \& Galindo, 2005; Herring \& Stoerger, 2013; Yates, 1997). 
A principio de los noventas cuando se iniciaron los primeros estudios en el campo de la CMC que incorporaron la variable de género, existía la esperanza que los foros online pudiesen incrementar la igualdad de género al permitir a los autores escoger seudónimos y a expresarse en un medio puramente textual, evitando las señales (lenguaje corporal, edad, raza, género, nivel socio-económico, etc.) que, en la comunicación cara a cara, ayudan a la formación de prejuicios sociales y estereotipos (Henrry, 2008; Morahan-Martin, 2000; Pierson, 2015).

Desafortunadamente estas expectativas no se cumplieron, los resultados de las primeras investigaciones -y hasta el día de hoy - sostienen que estas herramientas, lejos de estar contribuyendo a la plena incorporación y participación de las mujeres, refuerzan la desigualdad de género (Castaño, 2005; Sanchez, 2010; Yates, 1997). Esto, y en primer lugar, debido al menor acceso de las mujeres a Internet. Un estudio realizado por la Comisión Económica para América Latina y Caribe [CEPAL] (2013), dio a conocer que la tasa de uso de Internet de las mujeres es en promedio 8,5 por ciento menor a la de los hombres en 10 países. Y que pese a que las mujeres equiparan a los hombres en cuanto al acceso a Internet, presentan una clara desventaja con respecto a su uso.

En segundo lugar, e incluso cuando las mujeres logran equiparar a los hombres en cuanto a acceso, la igualdad de género sigue siendo un tema pendiente. Los trabajos de Henrry (2013) y Morahan-Martin (2000) dieron cuenta que las mujeres son expulsadas de los foros a través de tácticas agresivas y de acoso. Asimismo, las construcciones de género persisten en el espacio online: las mujeres tienden a ser más educadas, a hacer valer sus opiniones con menor fuerza que los hombres y a preferir productos acordes a los estereotipos de género.

Consecuentemente, se observó que los foros online reproducen los desequilibrios de poder observados en las interacciones offline. Un estudio realizado por Herring (1992) en un foro online perteneciente a la Sociedad Lingüística de América, dio cuenta que las mujeres estaban sub-representadas en relación a su participación en la sociedad y que los mensajes que publicaban eran más cortos y recibían menos respuestas, y que por el contrario, los hombres escribían comentarios más largos, eran capaces de defender con mayor fuerza sus opiniones, persistían con sus argumentos aun cuando sus comentarios no recibían respuestas, y que además, abrían y cerraban los debates.

Finalmente, y en el marco de los estudios de género en la prensa online, la publicación del estudio realizado por Pierson (2015) sobre la participación de las lectoras en los comentarios del The New York Times, dio a conocer que las mujeres sólo habían escrito el $25 \%$ los comentarios, pese a suponer el $44 \%$ del total de los lectores de ese medio. Y que ellas sólo escribían más comentarios que los hombres en aquellas publicaciones que trataban temas de paternidad y familia (79\% de participación), sobre bodas y moda (63\%), sobre cocina (53\%) y artículos escritos por otras mujeres.

Cabe destacar, que The New York Times, al igual que muchas otras plataformas web, utiliza un sistema de algoritmos que recomienda contenidos similares a los leídos con anterioridad, contribuyendo a perpetuar las diferencias y los estereotipos de género en el consumo y acceso a los contenidos. En este sentido, la autora del estudio señala que en una era donde el código impacta a millones de personas, los algoritmos tienen implicancias sociales no solo al reflejar la realidad, si no también, al darle forma.

\section{Marco Teórico}

\subsection{Categoría de género}

El género como una categoría de análisis nos plantea ineludiblemente una noción de relación, no es viable pensar en estudiar a las mujeres, en un mundo aislado de los hombres. Por este motivo, se emplea para nombrar las relaciones sociales entre sexos. Si bien el concepto género ha evolucionado a través del tiempo gracias a los aportes y reflexiones de las diversas disciplinas que lo han adoptado, se trabajó en base a la definición propuesta por la antropóloga Joan Scott (1990) donde género es un conjunto de elementos interrelacionados entre sí al interior de dos grandes proposiciones: el concepto género como un elemento constitutivo de las relaciones sociales basadas en las diferencias que distinguen los sexos, y como una forma primaria 
de relaciones de poder. El uso de este concepto lleva implícito el rechazo al determinismo biológico como explicación posible para la desigualdad, transformándose en "una forma de referirse a los orígenes exclusivamente sociales de identidades subjetivas de hombres y mujeres" (Scott, 1990, p. 28).

Por un lado, Amorós (1990) dirá que la dicotomía publico/privado- constituye una invariante estructural que articula las sociedades y jerarquizando los espacios tanto del hombre como de la mujer, y que esta división más allá de sus diferencias históricas presenta características que son recurrentes, a saber, las actividades socialmente más valoradas y las de mayor prestigio, las realizan prácticamente en todas las sociedades los hombres, y que estas son en su mayoría las que configuran el espacio público, el espacio del logos (Amorós, 1990).

Por otro lado, y a partir de la consigna "lo personal es político" Pateman (1996) sostiene, desde una postura feminista, que las circunstancias personales están estructuradas por factores públicos -por leyes sobre la violación y el aborto, por el estatus de esposas y con ello, las políticas de cuidado de los hijos y la división sexual del trabajo en el hogar y fuera de él-y que por lo tanto: "los problemas personales sólo se pueden resolver a través de medios y de acciones políticas" (16).

Una de las tantas prácticas que ha permitido perpetuar la estructura del dominio masculino en el espacio público y en los medios de comunicación, son las conductas sexistas y discriminatorias hacia las mujeres (Byerly \& Ross, 2006; Henrry, 2013; Morahan-Martin, 2000). La teoría de sexismo ambivalente propuesta por Glick y Fiske (1996), dio un marco para entender que tanto las actitudes benévolas como las hostiles son un mecanismo de coerción para aquellas mujeres que no cumplen con los estereotipos exigidos en las sociedades patriarcales (Cruz, Zempoaltecatl \& Correa, 2005), pudiendo de esta forma, obstaculizar su plena incorporación y participación en el espacio público.

\subsection{Comunicación y Género}

El informe MacBride (1980) señala que si bien los medios de comunicación social no son la causa fundamental de la subordinación de la mujer, ya que sus causas tienen profundas raíces en las estructuras sociales, políticas, económicas y culturales, estos tienen hasta cierto punto, la capacidad de estimular o retrasar ciertos cambios sociales. Además, se considera que son la plataforma inmejorable para el estudio de la variable de género, contribuyendo a eliminar prejuicios y estereotipos, aceptando el nuevo papel que le corresponde a la mujer dentro de la sociedad, y promoviendo su integración en el proceso de un desarrollo social equitativo (Franquet, Luzón \& Ramajo, 2006). De este modo, es posible señalar que los medios de comunicación:

aparecen como fines en sí, lugares influyentes de representación donde se pueden ofrecer imágenes y una sensibilidad nueva acerca de la igualdad entre los sexos, y poner de manifiesto la creatividad y las expresiones de las mujeres. Son también medios para alcanzar otro fines, vehículos destinados a facilitar el debate público sobre problemas y preocupaciones sociales más amplios (Sreberny-Mohammadi, 1996, p. 22).

En cuanto a los medios de comunicación online, la Red de Investigadoras por la vida y la Libertad de las mujeres (2008) señala que en este rubro y en general el de las nuevas tecnologías de la información y la comunicación, el diagnóstico al igual que en los medios tradicionales también es pesimista, ya que más que aportar a la plena incorporación de las mujeres en el espacio público, estas herramientas consolidan las desigualdades de género.

Por otro lado, la teoría de "comunicación y género en contextos públicos" desarrollado por las/ los autoras/es Pearson, Turner y Todd-Mancillas (1993) proporcionó un marco para comprender la participación de los lectores y las lectoras en los espacios de participación al lector. Esto, ya que los hechos apuntan a que existen ciertos comportamientos comunicativos de la comunicación cara a cara (CCC) que se extienden a la comunicación mediada por computadora (CMC) (Henrry, 2013; Morahan-Martin, 2000; Selfe \& Meyer, 1991). Entre los contextos públicos analizados por estos autores, determinaron que en las interacciones en grupos pequeños los hombres tienden a hablar más que las mujeres y que ellos son más propensos 
a iniciar las conversaciones y que tanto hombres como mujeres tienden a afiliarse al grupo que ostenta la mayoría. Sin embargo, las motivaciones entre ambos difieren: las mujeres suelen hacerlo cuando se sienten débiles, mientras que los hombres en el otro extremo, tienden a hacerlo cuando se sienten fuertes. Por otro lado, señalan que los individuos con mayor estatus son más eficaces comunicacionalmente que los que poseen un estatus inferior y que está la creencia que los hombres poseen un mayor estatus que el de las mujeres, siendo el acto de hablar en público una actividad tradicionalmente masculina.

\subsection{Participación en la prensa online}

De acuerdo a lo señalado por Martínez (2005) para comprender el concepto de participación, lo primero que se debe tener en cuenta es que la participación de los usuarios se define a partir de la interactividad: los usuarios interactúan entre sí, con los medios y los soportes virtuales, con los contenidos y con los emisores de esos contenidos.

Los medios online ofrecen a los usuarios diversas posibilidades de participación interactiva que van desde las funciones más reactivas de interacción con el medio (interacción sujeto/objeto) hasta otros modos de participación más interactivas de los usuarios con el medio, los contenidos y los periodistas, y de los usuarios entre sí (Martínez, 2005).

Raimondo (2012) propone una definición y una clasificación para aquellas instancias en la prensa online donde se materializa la actividad del lector, a saber, los espacios de participación del lector. Los espacios de participación del lector han sido aprovechados por los medios online para tomar el pulso de las opiniones de los lectores/as y como fuente de información, y a su vez, por los lectores, cómo vía para hacerse escuchar -por los medios y la propia audiencia-, destacando que

la adaptación de las ediciones digitales de la prensa tradicional a un modelo de periodismo más participativo ha evolucionado de manera paulatina. En mayor o menor medida, la mayoría de las ediciones digitales ofrecen hoy en día fórmulas que fomentan la incorporación de los ciudadanos a la esfera pública (Mendiguren, Pérez \& Peña, 2011, p. 423).

Los comentarios a las noticias, en cuanto a espacios de participación al lector, han sido incorporados por la prensa online en la mayoría de las noticias que publica, invitando al lector a opinar sobre los hechos y sucesos de la actualidad que son de interés público.

\section{Metodología}

Se diseñó una metodología en base a una investigación de tipo descriptiva, cuyo corpus-de formato documental- está compuesto por los comentarios de las lectoras y los lectores a las noticias publicadas en el diario digital Emol.com durante los meses de Septiembre y Noviembre del año 2015. También se consideraron aquellos comentarios que dentro de este contexto surgieran como respuesta a otros comentarios.

Emol.com provee un espacio para que los lectores comenten las noticias. Este espacio, al igual que los blogs, además de permitir una interacción dialógica, tienen una estructura que permite ver el desarrollo de un tema por varios integrantes. Su estructura de cascada se asemeja al de un diálogo cara a cara en el que los lectores y lectoras debaten en torno a una noticia (Arancibia \& Montecino, 2013). Además, por no tener un tope de palabras como Twitter u otras plataformas sociales, este espacio permite desarrollar argumentos en extenso como también admite la posibilidad de adjuntar videos y links a otras fuentes. Cabe señalar que Emol.com explicita en su sitio web que se guarda el derecho a no publicar las opiniones que considere agresivas y ofensivas, pudiendo filtrar los comentarios que allí se emiten.

La elección de este medio se debió principalmente a que Emol.com al momento de realizar el estudio era el quinto sitio web más visitado en Chile luego de Youtube.com, Google.cl, Facebook.com y Google.com ${ }^{1}$, y además, por ser considerado según el Primer Estudio Nacional sobre Lectoría de Medios Escritos realizado por la Universidad Diego Portales y el grupo de consultores Feedback (2010), como el medio de prensa online chileno más influyente, el que entrega información de mayor calidad 
Figura 1. Ficha de análisis.

\begin{tabular}{|c|c|}
\hline \multicolumn{2}{|c|}{ Ficha de Análisis no } \\
\hline 1. Área temática de la noticia: & 7. Sexo del autor del articulo: \\
\hline 2. Título de la noticia: & 8. Número de Comentarios en la publicación \\
\hline 3. Fecha de publicación: & Total Hombres: \\
\hline 4. Fecha de extracción: & Total Mujeres: \\
\hline 5. Fuente: & Total Indefinido: \\
\hline 6. Resumen de la noticia: & 9. Presencia de comentarios sexistas: \\
\hline \multicolumn{2}{|c|}{$\begin{array}{l}\text { 10. Comentarios: Traspaso del contenido de todos los comentarios, manteniendo el } \\
\text { mismo orden cronológico de la publicación y disposición de los textos. Los comentarios } \\
\text { que son respuesta a otros comentarios van marginados a la derecha. } \\
\text { a) Nombre del autor } \\
\text { b) Sexo del autor (hombre, mujer o indefinidol } \\
\text { b) Fecha y hora de publicación } \\
\text { c) Contenido de la Publicación }\end{array}$} \\
\hline
\end{tabular}

Fuente: Elaboración propia.

y el que cuenta con mayor credibilidad. En cuanto al perfil de su audiencia, el Informe de Lectoría de la Asociación Chilena de Agencias de Medios AG (2015) señaló que el 60\% de los lectores de Emol. com son hombres, mientras que el $40 \%$ son mujeres.

Para la construcción de la muestra, fueron inventariados en un archivo Word todos los documentos (enlaces de las noticias) que durante los meses que duró el estudio trataran los temas noticiosos establecidos en los objetivos. Luego, fueron seleccionados semanalmente y traspasados a una ficha de análisis aquellos documentos con el mayor número de comentarios en cada una de las categorías noticiosas.

Para analizar las frecuencias de los comentarios según variable sexo y categoría noticiosa, se trabajó en base a una muestra diversa o de máxima variación. Mientras que para establecer si las opiniones de los lectores y las lectoras expresaban distintos puntos de vista sobre un mismo asunto, se trabajó en base a una muestra intencionada compuesta por los comentarios a la noticia sobre la Ley de Despenalización del Aborto en sus tres causales $^{2}$ que, durante los meses que duró el estudio, fuese la noticia sobre este tema que tuviese el mayor número de comentarios. La elección de esta temática radicó en la preocupación de una sobre reprentación masculina en las opiniones sobre un asunto que afecta directamente a la mujer y los derechos sobre su cuerpo.
Para el análisis de los datos se recurrió al análisis de contenido que, dependiendo de cada objetivo, fue de orden cuantitativo, cualitativo o ambos. Con el fin de determinar la frecuencia de los comentarios según sexo y categoría noticiosa, se escogió como unidad de análisis los nombres de los autores de los comentarios, recordando que, el nombre del autor del comentario corresponde al nombre de su perfil de Facebook, por lo que se esperó dada la "política de nombres reales" de Facebook, trabajar en base a identidades reales. De este modo, los seudónimos, los nombres de fantasía y nombres falsos identificados fueron clasificados bajo la categoría de "indefinidos" y no se consideraron en la muestra.

La codificación se realizó en una instancia, se identificó el sexo de los autores de los comentarios en cada ficha de análisis, y se les asignó un color (rojo para los comentarios emitidos por mujeres, azul para los comentarios emitidos por hombres y verde para los indefinidos). El proceso de cuantificación se realizó en dos etapas: primero, se procedió a contabilizar el número de comentarios realizados por hombres, el número de comentarios realizados por mujeres y el número de comentarios realizados por "indefinidos" en cada una de las fichas. Luego, los datos de cada una de las fichas fueron traspasados, agrupados y cuantificados en un documento Excel, con el número de la ficha, las categorías (temas de los artículos) y sexo del autor del comentario.

Con los datos obtenidos en la matriz de contingen- 
Figura 2. Matriz de contingencia de codificación de datos.

\begin{tabular}{|c|c|c|c|c|c|c|c|c|c|c|c|c|c|}
\hline \multirow[b]{2}{*}{ FICHA DE ANÁLISIS } & \multicolumn{3}{|c|}{ POLÍtICA } & \multicolumn{3}{|c|}{ ECONOMÍA } & \multicolumn{3}{|c|}{ EDUCACIÓN } & \multicolumn{3}{|c|}{ CIENCIA Y TECNOLOGÍA } & \multirow{2}{*}{ TOTAL } \\
\hline & $\mathrm{H}$ & M & I & $\mathrm{H}$ & M & I & $\mathrm{H}$ & M & I & $\mathrm{H}$ & M & I & \\
\hline \begin{tabular}{|l|} 
Ficha 1 \\
\end{tabular} & 143 & 22 & 14 & & & & & & & & & & 179 \\
\hline Ficha 2 & & & & 154 & 3 & 6 & & & & & & & 163 \\
\hline Ficha 3 & & & & & & & 49 & 2 & 10 & & & & 61 \\
\hline Ficha 4 & & & & & & & & & & 47 & 2 & 3 & 52 \\
\hline Ficha 5 & 97 & 14 & 6 & & & & & & & & & & 117 \\
\hline Ficha 6 & & & & 88 & 1 & 3 & & & & & & & 92 \\
\hline Ficha 7 & & & & & & & 0 & 0 & 0 & & & & 0 \\
\hline Ficha 8 & & & & & & & & & & 6 & 0 & 1 & 7 \\
\hline Ficha 9 & 168 & 29 & 44 & & & & & & & & 4 & & 241 \\
\hline Ficha 10 & & & & 11 & 1 & 1 & & & & & & & 13 \\
\hline Ficha 11 & & & & & & & 61 & 9 & 7 & & & & 77 \\
\hline Ficha 12 & & & & & & & & & & 44 & 3 & 4 & 51 \\
\hline Ficha 13 & 21 & 1 & 0 & & & & & & & & & & 22 \\
\hline Ficha 14 & & & & 87 & 4 & 11 & & & & & & & 102 \\
\hline Ficha 15 & & & & & & & 35 & 4 & 5 & & 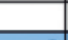 & & 44 \\
\hline Ficha 16 & & & & & & & & & & 73 & 3 & 5 & 81 \\
\hline Ficha 17 & 18 & 1 & 1 & & & & & & & & 4 & & 20 \\
\hline Ficha 18 & & & & 66 & 0 & 3 & & & & & & & 69 \\
\hline Ficha 19 & & & & & & & 52 & 9 & 5 & & & & 66 \\
\hline Ficha 20 & & & & & & & & & & 36 & 0 & 2 & 38 \\
\hline Ficha 21 & 209 & 8 & 13 & & & & & & & & & & 230 \\
\hline Ficha 22 & & & & 62 & 0 & 4 & & & & & & & 66 \\
\hline Ficha 23 & & & & & & & 10 & 1 & 4 & & & & 15 \\
\hline Ficha 24 & & & & & & & & & & 5 & 0 & 0 & 5 \\
\hline Ficha 25 & 49 & 16 & 13 & & & & & & & & & & 78 \\
\hline Ficha 26 & & & & 34 & 2 & 2 & & & & & & & 38 \\
\hline Ficha 27 & & & & & & & 37 & 6 & 5 & & & & 48 \\
\hline Ficha28 & & & & & & & & & & 37 & 2 & 9 & 48 \\
\hline Ficha29 & 54 & 1 & 5 & & & & & & & & & & 60 \\
\hline Ficha 30 & & & & 83 & 4 & 2 & & & & & & & 89 \\
\hline Ficha 31 & & & & & & & 41 & 8 & 4 & & & & 53 \\
\hline Ficha 32 & & & & & & & & & & 1 & 0 & 0 & 1 \\
\hline Ficha 33 & 49 & 10 & 5 & & & & & & & & & & 64 \\
\hline Ficha 34 & & & & 69 & 6 & 4 & & & & & & & 79 \\
\hline Ficha35 & & & & & & & 61 & 8 & 3 & & & & 72 \\
\hline Ficha 36 & & & & & & & & & & 5 & 0 & 0 & 5 \\
\hline Ficha 37 & 61 & 6 & 1 & & & & & & & & & & 68 \\
\hline Ficha 38 & & & & 16 & 2 & 0 & & & & & & & 18 \\
\hline Ficha 39 & & & & & & & 61 & 3 & 3 & 1 & & & 67 \\
\hline Ficha 40 & & & & & & & & & & 0 & 0 & 0 & 0 \\
\hline \begin{tabular}{|l|} 
Ficha 41 \\
\end{tabular} & 13 & 3 & 3 & & & & & & & & & & 19 \\
\hline Ficha 42 & & & & 49 & 8 & 7 & & & & & & & 64 \\
\hline Ficha 43 & & & & & & & 78 & 13 & 7 & & & & 98 \\
\hline \begin{tabular}{|l|} 
Ficha 44 \\
\end{tabular} & & & & & & & & & & 4 & 0 & 1 & 5 \\
\hline Ficha 45 & 119 & 8 & 3 & & & & & & & & & & 130 \\
\hline Ficha 46 & & & & 72 & 0 & 1 & & & & & & & 73 \\
\hline Ficha 47 & & & & & & & 32 & 4 & 6 & & & & 42 \\
\hline \begin{tabular}{|l|} 
Ficha 48 \\
\end{tabular} & & & & & & & & & & 46 & 0 & 2 & 48 \\
\hline \begin{tabular}{|l|} 
Ficha 49 \\
\end{tabular} & 103 & 15 & 9 & & & & & & & & & & 127 \\
\hline Ficha 50 & & & & 123 & 5 & 13 & & & & & & & 141 \\
\hline Ficha 51 & & & & & & & 20 & 3 & 1 & & & & 24 \\
\hline Ficha 52 & & & & & & & & & & 7 & 0 & 2 & \\
\hline
\end{tabular}

\begin{tabular}{|c|c|c|c|c|c|c|c|c|c|c|c|c|c|}
\hline & $\mathrm{H}$ & M & I & $\mathrm{H}$ & M & I & $\mathrm{H}$ & M & & $\mathrm{H}$ & M & I & \\
\hline TOTAL & 1104 & 134 & 117 & 914 & 36 & 57 & 537 & 70 & 60 & 311 & 10 & 29 & $H+M+I$ \\
\hline TOTAL & & 1355 & & & 1007 & & & 667 & & & & 350 & 3379 \\
\hline
\end{tabular}

cia se calcularon las frecuencias absolutas (marginales) según sexo y categoría noticiosa, luego las frecuencias relativas totales $(\mathrm{fi} .=\mathrm{ni} / \mathrm{N})$ según la variable de sexo, y las frecuencia relativas conjuntas, es decir, el cociente entre la frecuencia abso- luta de comentarios observados en cada uno de los atributos de la variable "sexo" en conjunto a cada uno atributos de la variable "categorías" (fij=nij/n $x$ 100).

Para analizar si las lectoras y los lectores expre- 
Figura 3. Tabla de distribución de frecuencias relativas conjuntas de los comentarios emitidos según sexo del autor y categoría noticiosa.

\begin{tabular}{|c|c|c|c|c|c|}
\hline & \multicolumn{4}{|c|}{ TOTAL COMENTARIOS SEGÚN CATEGORÍAS (\%) } \\
\hline & & POLITICA & ECONOMÍA & EDUCACIÓN & $\begin{array}{c}\text { CIENCIAY } \\
\text { TECNOLOGIA } \\
\end{array}$ \\
\hline $\begin{array}{l}\bar{o} \\
\stackrel{0}{\circ} \\
\stackrel{x}{4} \\
z\end{array}$ & 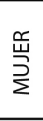 & $11 \%$ & $4 \%$ & $12 \%$ & $3 \%$ \\
\hline 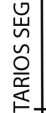 & 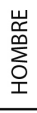 & $89 \%$ & $96 \%$ & $88 \%$ & $97 \%$ \\
\hline 己. & 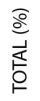 & $100 \%$ & $100 \%$ & $100 \%$ & $100 \%$ \\
\hline
\end{tabular}

Fuente: Elaboración propia.

saban distintos puntos de vista con respecto a un mismo tema, las unidades de análisis correspondieron a las todas las frases o párrafos donde los autores de los comentarios expresaron su punto de vista sobre la legislación del aborto. Para la definición de las categorías, se recurrió al proyecto de Ley que regula la despenalización del aborto en tres causales, a saber: peligro para la vida de la mujer, inviabilidad fetal de carácter letal y embarazo por violación. Por consiguiente, las catego- rías de análisis diseñadas fueron: "a favor" cuando la unidad de análisis expresó un punto de vista a favor de una o más causales de la ley de aborto, "ambivalente" para los casos en donde se expresó un punto de vista a favor de una o dos causales y en contra de una o dos causales, y finalmente "en contra" cuando el punto de vista expresó estar en contra de una o más causales de la ley de aborto. Una vez enumerados todos los segmentos, se procedió a clasificar las unidades del análisis en una matriz de contingencia de codificación de unidades de análisis, elaborada con los segmentos encontrados, categorías y sexo del autor del comentario.

Una vez que fueron traspasadas y clasificadas todas las unidades de análisis,se contabilizaron la cantidad de registros (opiniones) observados según el sexo del autor, para luego calcular las frecuencias relativas totales (marginales). Posteriormente, se procedió a contabilizar la cantidad de registros en cada categoría según sexo del autor a modo de identificar las opiniones predominantes en ambos casos (hombres y mujeres). Finalmente, y con los datos obtenidos en la tabla de distribución de frecuencias absolutas se calculó la frecuencia relativa conjunta.

Figura 4. Extracto matriz de contingencia de codificación de unidades de análisis.

\begin{tabular}{|c|c|c|c|c|c|c|}
\hline \multirow{2}{*}{$\begin{array}{l}\text { Nombre autor } \\
\text { del Comentario }\end{array}$} & \multirow{2}{*}{ Unidad de Análisis (Comentario) } & \multicolumn{2}{|c|}{ SEXO } & \multirow[b]{2}{*}{ A FAVOR } & \multirow[b]{2}{*}{ EN CONTRA } & \multirow[b]{2}{*}{ AMBIVALENTE } \\
\hline & & $\mathrm{H}$ & M & & & \\
\hline 1.Ignacio Dlaz de Valdés & Bien por Chile, una deuda social, bien acotada y donde todos los sectores fueron escuchados. & $\mathbf{\square}$ & & - & & \\
\hline 2. Raul Hernandez & $\begin{array}{l}\text { Realmente dan risa los comentarios de muchas personas, el proyecto permititía la interrupción del embarazo en tres } \\
\text { causalest: vivolación, inviabilidad fetal y riesgo de vida de la madre. Ahora este primero es el que aun se encuentra en } \\
\text { debate, por ser un tema mas moral que los otros } 2 \text {, porque en ese caso no hay problemas médicos que lo justifiquen. Si } \\
\text { se logra aprobar ese punto que es el mas causa controversia, dependerá de los valores de cada uno, si los moralistas } \\
\text { conservadores que se creen dueños de la verdad según sus creencias religiosas, que en estos temas importan menos } \\
\text { que un pepino, pero para los otros } 2 \text { esta totalamente justificado y si dicen que es asesinato, matar y aborto libre, no } \\
\text { tienen idea de lo que significa inviabilidad intrauterina y extrauterina. Lean mas ciencia y menos biblia, o sino no vayan } \\
\text { al medico y muchos otros ejemplos que les puedo dar a estos pseudo religiosos doble estándar }\end{array}$ & $\mathbf{a}$ & & $\odot$ & & \\
\hline 3.Jván Fernández & $\begin{array}{l}\text { Un gran paso hacia la decadencia moral y el total desprecio por la vida?, Hitler y el Dr. Mengele deben estar revolcándo- } \\
\text { se en sus tumbas de pura felicidad, hay en Chile partidarios al igual que ellos, de eliminar mediante feticidio a los } \\
\text { malformados o con sindrome de Dawn. }\end{array}$ & $\mathbf{\square}$ & & & ○ & \\
\hline 4. Isidora Alvarez Roll & $\begin{array}{l}\text { Que crimen ??... que pena vivir en un pais asill.... cuando una mamá está embarazada hay DOS PERSONAS con derechos. } \\
\text { No sé que clase de ética aprendieron los doctores que lo hacen, y que entienden como fin de la medicina. }\end{array}$ & & $\mathbf{\square}$ & & ○ & \\
\hline 5. Ana María Díaz & $\begin{array}{l}\text { Que sabe Monckeber sobre lo que daña a una mujer. Quitarle los derechos sobre su cuerpo es dañarla. Un monton de } \\
\text { patriarcales tratando de seguir controlando a la mujer a través de su cuerpo. }\end{array}$ & & $\mathbf{\square}$ & 0 & & \\
\hline 6. María Paz Castillo Mejías & No acostumbro hablar asi, pero la noticia lo amerita: -"hijos de puta los que aprobaron el asesinato!II"- & & $\mathbf{\square}$ & & - & \\
\hline 7. Franklin Avila & Me parece bien, ahora ojala que se apruebe el aborto por violación. & $\mathbf{\square}$ & & - & & \\
\hline 8. Solia Muñoz & $\begin{array}{l}\text { EL MISMO ADOLF HITLER FOMENTABA QUE LAS MUJERES ALEMANAS TUVIERAN HUOS Y ACA HOY SE ORDENA POR LEY } \\
\text { ASESINARLOS EN EL VIENTRE. POLITICOS COBARDES, CANALLAS, ASESINOS QUE SE LES PUDRAN LAS MANOS POR } \\
\text { FIRMAR TAL CRIMEN. }\end{array}$ & & $\mathbf{a}$ & & 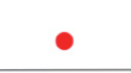 & \\
\hline 9. Danilo Silva Fierro & $\begin{array}{l}\text { Muy mal por Chile, una minoria corrupta que se autoproclama "nueva mayoria" - que en si no existe- con una presiden- } \\
\text { ta corrupta, perdida y sin rumboy que nadie la quiere en el poder deciden legalizar el asesinato de bebes no dados a } \\
\text { luz; con frases panfletarias, slogans mentirosos y manipulación mediática legalizan el asesinato, ellos son los que bien } \\
\text { describe el Libro de Romanos en su capitulo } 1 \text {. Dios tenga misericordia. }\end{array}$ & $\mathbf{\square}$ & & & - & \\
\hline 10. Franklin Avila & $\begin{array}{l}\text { En este caso no se trata de una minoría, porque sobre el } 70 \% \text { de las personas estamos a favor del aborto en las } 3 \\
\text { causales; muy distinto es el tema de que la madame tiene el } 24 \% \text { de apoyo. }\end{array}$ & $\mathbf{a}$ & & ○ & & \\
\hline 11. Samuel Enrique & $\begin{array}{l}\text { Acevedo Esto ya es una Burla... ya es Terrible que aprueben la despenalizacion del Aborto... pero mas terrible es que } \\
\text { Cualquier Medico pueda realizar un Aborto!I... Esta claro que buscan el Aborto Libre... porque nunca podran controlar } \\
\text { todo el Universo de Médicos... una Burla.... Hay leyes que Protegen el Maltrato Animal.... pero no hay leyes que protejan } \\
\text { a nuestro Niños....... Chile Pais de Caretas!.... }\end{array}$ & $\mathbf{a}$ & & & - & \\
\hline
\end{tabular}

Fuente: Elaboración propia. 
Figura 5. Tabla de distribución de frecuencias relativas conjuntas según sexo y subcategorías analizadas sobre la Ley del aborto.

\begin{tabular}{|l|c|c|c|c|}
\cline { 2 - 4 } \multicolumn{1}{c|}{} & \multicolumn{3}{c|}{ OPINIONES SOBRE LA LEY DE ABORTO } & \multicolumn{1}{c}{} \\
\cline { 2 - 4 } \multicolumn{1}{c|}{} & A FAVOR & EN CONTRA & AMBIVALENTE & TOTAL \\
\hline HOMBRE & $53 \%$ & $46 \%$ & $1 \%$ & $100 \%$ \\
\hline MUJER & $27 \%$ & $73 \%$ & - & $100 \%$ \\
\hline
\end{tabular}

Fuente: Elaboración propia.

Finalmente, para identificar y cuantificar la presencia de discursos sexistas al interior de los comentarios también se recurrió al análisis de contenido de tipo cuantitativo y cualitativo. Las unidades de análisis correspondieron a todas las palabras, frases, párrafos o el comentario en su totalidad, que presentaron conceptos sexistas en los comentarios que conformaron la muestra. Las categorías y subcategorías se definieron de acuerdo a las variables teóricas del concepto de sexismo ambivalente. De este modo, las unidades de análisis fueron extraídas, clasificadas y cuantificadas según el sexo del autor, la categoría y subcategoría dentro del sexismo ambivalente.

\section{Resultados}

\subsection{Frecuencias de los comentarios según sexo del autor y tema del artículo}

A partir de la metodología propuesta, fueron recuperados de Emol.com un total de 3.379 documentos (comentarios), de los cuales fueron excluidos de la muestra 263 por tratarse de comentarios cuyo sexo del autor se identificó como "indefinido". De esta forma, la muestra del estudio quedó conformada por un total de 3.116 comentarios, de los cuales $1.238(40 \%)$ fueron emitidos en la sección de política, 950 (30\%) en la sección de economía, $607(20 \%)$ en educación y de $321(10 \%)$ en ciencias y tecnología.

Del total de casos observados (comentarios) en los que pudo ser identificado el sexo del autor, sólo 250 comentarios (8\%) fueron escritos por mujeres, mientras que 2.866 (92\%) fueron escritos por hombres.

En cuanto a los niveles de participación según categoría noticiosa, se observaron algunas variaciones según el tema. Se constató que las categorías donde hubo una mayor participación de las mujeres fue en los comentarios a las noticias sobre política y educación. En política, ellas escribieron el 11\% de los comentarios, mientras que en educación la cifra aumenta ligeramente al $12 \%$. En contraste, los hombres emitieron el $89 \%$ de los comentarios a las noticias sobre política y el $88 \%$ en educación.

En las categorías noticiosas de economía y ciencias y tecnología la participación de las mujeres disminuye considerablemente. En economía, ellas tan

Figura 6. Matriz de contingencia de codificación de unidades de análisis.

\begin{tabular}{|c|c|c|c|c|c|c|c|}
\hline \multirow{2}{*}{\multicolumn{2}{|c|}{ UNIDAD DE ANÁLISIS }} & \multicolumn{3}{|c|}{ SEXISMO BENÉVOLO } & \multicolumn{3}{|c|}{ SEXISMO HOSTIL } \\
\hline & & $\begin{array}{l}\text { PATERNALISMO } \\
\text { PROTECTOR } \\
\end{array}$ & \begin{tabular}{|l|} 
DIFERENCIACIÓN \\
COMPLEMENTARIA \\
\end{tabular} & \begin{tabular}{|l|} 
INTIMIDAD \\
HETEROSEXUAL \\
\end{tabular} & $\begin{array}{l}\text { PATERNALISMO } \\
\text { DOMINADOR }\end{array}$ & $\begin{array}{l}\text { DIFERENCIACIÓN } \\
\text { COMPETITIVA }\end{array}$ & $\begin{array}{l}\text { DOMINACIÓN } \\
\text { HETEROSEXUAL } \\
\end{array}$ \\
\hline (1) & "Keep dreaming pussyUDlondo" & & & & & & $\mathrm{H}$ \\
\hline (2) & "iii Pobrecita tù ninitita !!!" & & & & $\mathrm{H}$ & & \\
\hline (3) & "Anda a enterrarte vieja menopáusica" & & & & & $\mathrm{H}$ & \\
\hline (4) & $\begin{array}{l}\text { "Anda a ver que estas quemando las } \\
\text { camisas conla plancha." }\end{array}$ & & $\mathrm{H}$ & & & & \\
\hline (5) & "Vaya a cocina" & & $\mathrm{H}$ & & & & \\
\hline (6) & "Claudita usted es bonita y rubia ? Jajaja" & & & & & & $\mathrm{H}$ \\
\hline (7) & "Vieja Q.LI.A" & & & & & & $\mathrm{H}$ \\
\hline (8) & "Yo te puedo arreglar el pasaporte..." & $\mathrm{H}$ & & & & & \\
\hline & $\begin{array}{l}\text { "Natalia Sofia a a mejor tienes razón Natalie } \\
\text { (hermosa canción de Gilbert Becaud)." }\end{array}$ & & & $\mathrm{H}$ & & & \\
\hline$(10)_{e}$ & $\begin{array}{l}\text { "Tanto tiempo q se toma sra para } \\
\text { escribir tonterass!!!" }\end{array}$ & & & & & $\mathrm{H}$ & \\
\hline
\end{tabular}


Figura 7. Participación según sexo y categoría noticia.

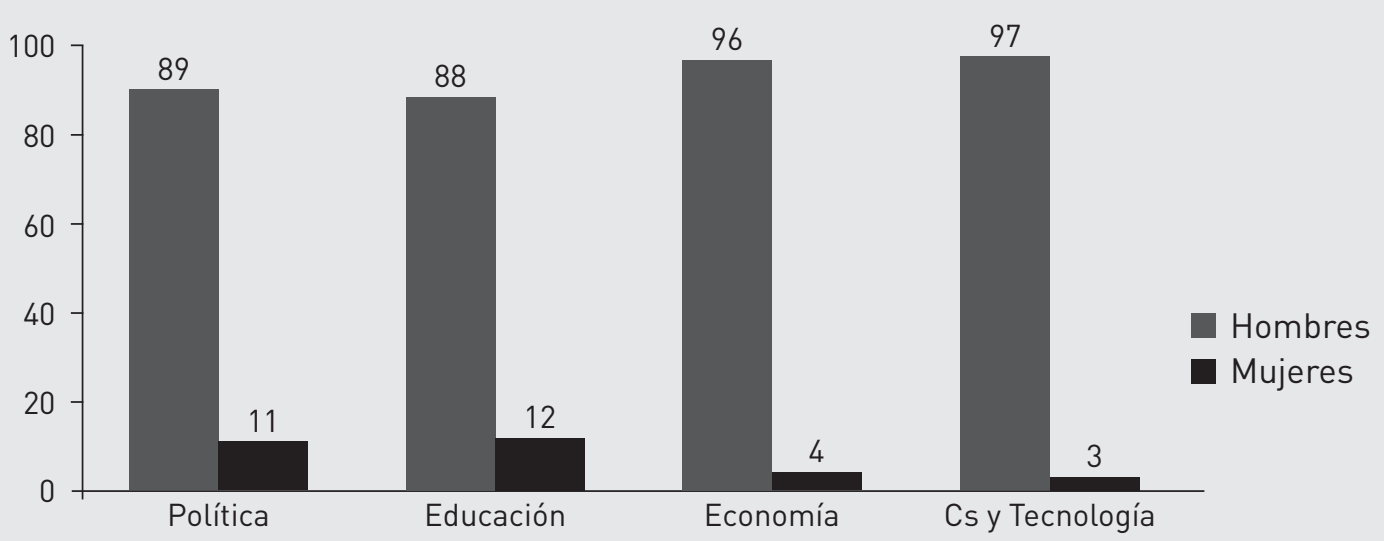

Fuente: Elaboración propia.

sólo escribieron el $4 \%$ de los comentarios mientras en que en ciencia y tecnología este porcentaje desciende al 3\%. Esta participación marginal de las mujeres contrasta con la de los hombres, donde ellos escribieron el $96 \%$ de los comentarios a las noticias en economía y el $97 \%$ de los comentarios en ciencias y tecnología.

El leve aumento de los comentarios emitidos por la mujeres a las noticias sobre educación, reflejaría la experiencia cotidiana de las mujeres que confirma la separación entre lo público y lo privado, al constatar que las mujeres tienden a participar un poco más en en la medida que se abordan temas propios del espacio privado, siendo en este caso, la discusión sobre la educación de los hijos.

\subsection{Puntos de vista expresados en los comentarios según sexo del autor}

En base a la metodología propuesta, de un total de cuatro publicaciones sobre la Ley de despenalización del aborto durante el intervalo de tiempo definido para la extracción de la muestra, se utilizó para el análisis la noticia publicada el día 8 de septiembre en la cual se daba a conocer la aprobación de la despenalización del aborto en dos de sus tres causales $^{3}$, donde, de un total 415 comentarios extraídos, se pudo constatar que 283 fueron escritos por hombres y 97 por mujeres, representando estos últimos un $23 \%$ del total.
Figura 8. Imagen de la noticia extraída el 8 de septiembre del 2015 desde Emol.com

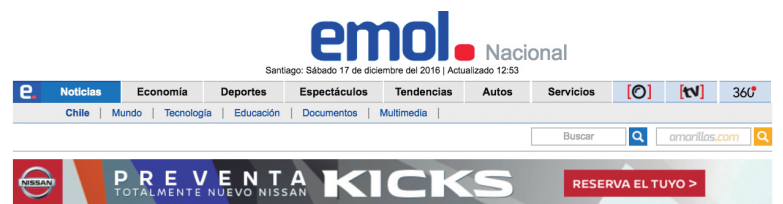

Aborto: Se aprueba despenalización por mal formación y riesgo de vida de la madre

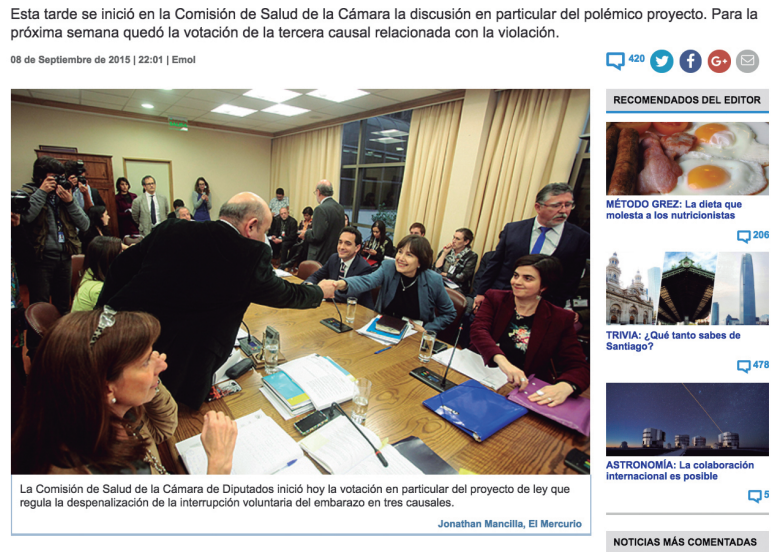

Fuente: Emol.com

De los 380 comentarios escritos por hombres y mujeres, solo 223 de ellos expresaron un punto de vista (directa o indirectamente) sobre la Ley de despenalización del aborto en tres causales. De estos, $156(70 \%)$ corresponden a comentarios escritos por hombres y $67(30 \%)$ escritos por mujeres. De este modo, se da cuenta de una clara sobre-representación masculina en un tema que afecta directamente a la mujer y los derechos sobre su cuerpo. 
Figura 9. Puntos de vista expresados sobre la ley de despenalización del aborto en tres causales según sexo del autor.

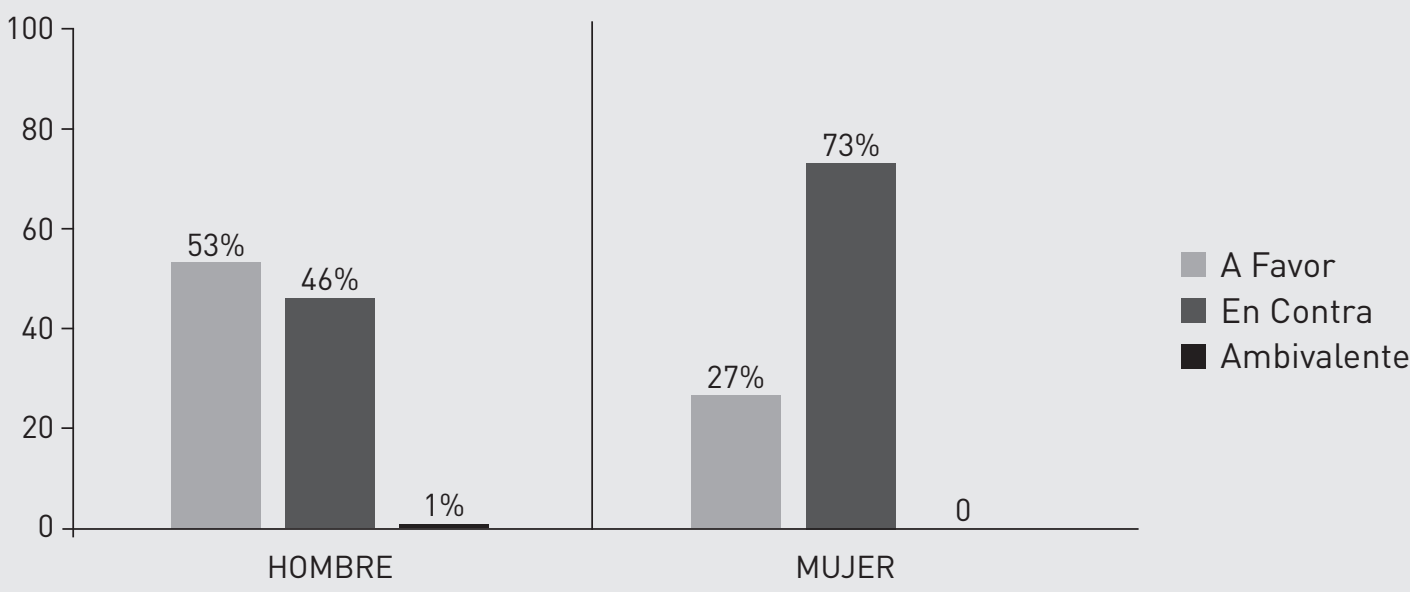

Fuente: Elaboración propia.

Ahora bien, en base a las categorías establecidas en la metodología la favor, en contra y ambivalente), de los 156 comentarios emitidos por hombres donde fue posible reconocer y determinar el punto de vista del autor del comentario, se estableció que el $53 \%$ expresó estar a favor de una o más causales de la ley de aborto, el $1 \%$ mostró estar a favor de una o dos causales y en contra de una o dos causales y el $46 \%$ estar en contra de una o más causales de la ley de aborto. Por el contrario, de los 67 comentarios emitidos por mujeres, se constató que sólo el $27 \%$ está a favor de una o más causales de la ley de aborto mientras que un $73 \%$ expresó estar en contra de una o más causales de la ley de aborto, en el caso de las mujeres no se observaron puntos de vistas ambivalentes.

De acuerdo a los datos observados en las categorías "a favor" y "en contra" según variable de sexo, es posible constatar que en el caso de los hombres no se aprecia un gran desequilibrio en la distribución de los puntos de vista expresados. Por el contrario, en los puntos de vista expresados por las mujeres las diferencias son evidentes, la amplia mayoría de ellas se muestra en contra de la Ley, dando cuenta que la mayoría de las opiniones expuestas responden a posiciones pro-vida 0 a roles de género femeninos conservadores. Finalmente, se estableció que los puntos de vista de las lectoras y los lectores sobre esta Ley difieren considerablemente, mientras que la mayoría de los hombres se muestran a favor (53\%) inversamente las mujeres expresan estar en contra (73\%) de la Ley por la despenalización del aborto en tres causales.

\subsection{Discursos Sexistas}

A partir del planteamiento metodológico, de los de 3.116 casos observados (comentarios) que compusieron la muestra del estudio, se identificó la presencia de discursos sexistas en 9 de ellos, emitidos en su totalidad por hombres, representando marginalmente $0,2 \%$ del total de la muestra. Cabe destacar que el bajo número de discursos sexistas observados podría deberse a las normas éticas del medio, es decir, Emol.com tiene la facultad de eliminar aquellos comentarios que considere ofensivos o agresivos. Los lectores y las lectoras también pueden denunciar a través de una herramienta que proporciona este sitio aquellos comentarios que estimen ofensivos o spam.

El $60 \%$ de de estos discursos se concentraron en la categoría de "Sexismo Hostil", frente al $40 \%$ en la categoría de "Sexismo Benevolente". En la categoría de "Sexismo Hostil" se presenciaron discursos sexistas en todas las subcategorías, siendo "Dominación Heterosexual" aquella subcategoría en la que se evidenció el mayor número de casos. Cabe recordar que la dominación sexual, se refiere a la hostilidad hacia las mujeres asociadas a la creencia de que ellas usarían su atractivo sexual para dominar a los hombres y que utilizarían el sexo, visto popularmente como un recurso del que ellas son guardianas, para manipular a los hombres.

Por otro lado, se identificó que el $89 \%$ de los discursos sexistas se concentraron en los comentarios de las noticias sobre política -segunda categoría 
con mayor participación de las lectoras- mientras que el $11 \%$ restante se realizó en los comentarios a las noticias sobre economía. Estos hallazgos si bien son esperanzadores, la baja presencia de comentarios sexistas en los espacios de participación al lector/a no debe minimizar la importancia de aquellos que sí fueron emitidos, ya que se estaría invisibilizando la presencia de un mecanismo de coerción que busca perpetuar la estructura del dominio masculino dentro del espacio público, y que justifica su poder (el de los hombres) a partir de la supuesta inferioridad o diferencia de las mujeres (Loscertales \& Fernández, 2009).

\section{Conclusiones}

Los resultados de este estudio demuestran cómo las diferencias de género se expresan en las interacciones y niveles de participación, ya que son mayoritariamente hombres quienes se apoderan de este espacio virtual para expresar sus opiniones, siendo principalmente masculino el territorio virtual de las interacciones en las categorías noticiosas analizadas en este medio. De este modo, se demuestra la subrepresentación de las mujeres en estos espacios virtuales, donde ellas prefieren omitir sus opiniones frente a la enorme cantidad de comentarios masculinos.

Estos resultados no solo podrían ser un reflejo de amplia desigualdad de género que existe en los medios de comunicación, sino también, de la escasa participación de las mujeres en el espacio público, cuyo acceso ha estado obstaculizado por los valores y tradiciones de una estructura patriarcal que históricamente ha desvalorizado el lugar de la mujer en la sociedad, relegándola al espacio privado y limitando su rol social a su papel reproductor -ámbito doméstico, crianza de los hijos y la reproducción cotidiana- (Otner, 1979).

Por otro lado, se presume que el limitado acceso de las mujeres a los centro de poder, así como a las instituciones públicas y a los gabinetes, también podría ser un factor que desmotivaría a las mujeres a participar en estos espacios, ya que al menos en las categorías noticiosas analizadas (política, economía, ciencias y tecnología y educación), estos temas siguen siendo "de hombres y para hombres. La baja participación de las mujeres en estos espacios ilustra la desfavorable situación de las mujeres en los medios de comunicación en términos de igualdad y de representación. En primer lugar, la sub-representación femenina observada implica que las pocas mujeres que opinan no estarían reflejando de forma significativa lo que piensa la mayoría, repercutiendo en una adecuada representación de sí mismas. Igualmente, las opiniones emitidas (de hombres y mujeres) reflejarían de forma equitativa los puntos de vista que allí se exponen (Pierson, 2015).

A partir de la consigna “lo personal es político ( $\mathrm{Pa}-$ teman, 1996), la sub-representación de las mujeres observada en los comentarios a la noticia sobre aborto, implica que las lectoras de este medio siguen percibiendo el aborto como un tema privado, cuando por el contrario se espera que al ser un tema privado configurado por factores públicos, estos temas sean resueltos a través de medios y de acciones políticas. En este sentido, se releva la importancia de internet como una herramienta esencial para la dinámica social, ya que tiene la capacidad de legitimar los discursos y generar reconocimiento social.

Es importante señalar que el escaso número de comentarios sexistas observados $(0,2 \%)$ en los espacios de participación al lector/ar no debe minimizar la importancia de aquellos que sí fueron emitidos, dado a que su mera presencia indicaría que siguen operando estas conductas coercitivas que buscan perpetuar la estructura del dominio masculino, reduciendo a las mujeres y por lo tanto, entorpeciendo su plena participación en los medios de comunicación, donde la comunicación como un derecho humano debe contemplar el pleno, libre, amplio e indisputable acceso de las mujeres a la comunicación por cualesquiera medios (Vega, 2010).

Es posible concluir que pese a la existencia de nuevas herramientas de participación en la prensa online, aún está pendiente transformar estos espacios en lugares de diálogo democrático de ideas y pensamientos, que permitan enriquecer el debate público sobre asuntos sociales y políticos y, asimismo, fomentar la participación de los ciudadanos/as en asuntos públicos.

Se espera que los resultados de este estudio abran nuevas interrogantes y líneas de investigación que 
indaguen sobre las causas de este fenómeno, así como exploren mecanismos que contribuyan a fortalecer y fomentar la participación y el acceso de las mujeres a los medios de comunicación ya sea como productoras, usuarias o usuarias-productoras, dada las implicancias de los medios online e Internet en la promoción de la mujer. Sobre todo hoy, en un contexto social como el chileno, que exige mayor equidad, justicia y representatividad y donde el movimiento feminista ha dado un salto sustancial en el reconocimiento de sus demandas, gracias a las capacidades de organización y protagonismo que otorga Internet y los medios digitales (Burch, 1996).

\section{Notas}

1. Información entregada por el sitio de Amazon Alexa.com (2016)

2. En septiembre de 2017 fue promulgada la ley $N^{\circ} 21.030$ que regula la despenalización de la interrupción voluntaria del embarazo en tres causales: peligro para la vida de la mujer, inviabilidad fetal de carácter letal y embarazo por violación.

3. Se aprueba despenalización del aborto por mal formación del feto y riesgo de vida de la madre. (2015, Septiembre 8). Emol.com. Recuperado de http://www. emol.com/noticias/Nacional/2015/09/08/748937/ Aborto-Se-aprueba-despenalizacion-por-malformacion-y-riesgo-de-vida-de-la-madre.html

\section{Referencias}

Asociación Chilena de Agencia de Medios AG. (2105). Informe de Lectoría. Recuperado de http://aam.cl/wp-content/uploads/2018/05/Informe_de_lectoria_AAM.pdf

Arancibia, M. \& Montecino, L. (2010). El blog de comentarios a textos de opinión en ciberperiódicos: un género en constante reconstrucción. Representaciones discursivas sobre la pobreza en ciberperiódicos chilenos.. Literatura y Lingüística, 28, $123-148$.

Amorós, C. (1990). Espacio público, espacio privado y definiciones ideológicas de" lo masculino" y “lo femenino". En Participación, cultura política y Estado. Buenos Aires: Ediciones de la Flor.

Burch, S. (1996). El reto de las nuevas tecnologías. En: Por todos los medios: comunicación y género. Portugal, A y Torres, C. (ed). pp 17-35. Edición de las Mujeres. № 23. Isis Internacional.

Byerly, C. \& Ross, K. (2006). Women and Media. A Critical Introduction. Londres: Blackwell.

Castaño, C. (2005). Las mujeres y las tecnologías de la información. Internet y la trama de nuestra vida. Madrid: Alianza Editorial.

Cruz, C., Zempoaltecatl, V. \& Correa, F. (2005). Perfiles de sexismo en la ciudad de México: validación del cuestionario de medición del sexismo ambivalente. Enseñanza e Investigación en Psicología, (10) 2, julio-diciembre, 381-395. Recuperado de http://www.redalyc.org/articulo.oa? $\mathrm{id}=29210212$

Franquet, R., Luzón, V. \& Ramajo, N. (2006). Mujer y medios de comunicación online. Un análisis de género. Departamento de Comunicación Audiovisual y Publicidad de la Universidad Autónoma de Barcelona.

Gómez, E. \& Galindo,A. (2005). Los Estudios de Comunicación Mediada por Computadora: una Revisión y algunos Apuntes. Razón y Palabra, (10) 44, pp. 1-16. 
Herring, S. (1992). Gender and Participation in Computer-Mediated Linguistic Discourse. Paper presented at the Annual Meeting of the Linguistic Society of America (Philadelphia, PA, January 9-12, 1992). Recuperado de https://files.eric.ed.gov/ fulltext/ED345552.pdf

Herring, S. (2000). Gender differences in CMC: Findings and implications. Computer Professionals for Social Responsibility Journal (18) 1. Recuperado de https://www.researchgate.net/profile/Susan_Herring3/publication/246291970_Gender_Differences_in_CMC_Findings_and_Implications/links/55453b2c0cf24107d397b0e5/ Gender-Differences-in-CMC-Findings-and-Implications.pdf

Herring, S. (2008). Gender and Power in On-line Communication. In J.Holmes, M. Meyerhoff \& S. Ehrlich (eds.), Handbook of Language and Gender. Hoboken, NJ:WileyBlackwell Publishing, pp. 202 - 228.

Herring, S. \& Stoerger, S. (2013). Gender and (A)nonymity in computer-mediated communication 2nd edition. In J.Holmes, M. Meyerhoff \& S. Ehrlich (eds.), Handbook of Language and Gender. Hoboken, NJ:Wiley-Blackwell Publishing, pp. 567 - 586.

Loscertales, F,. Fernández, E. y Higazi, Z. (2006). Violencia contra las mujeres en Isos de medios de comunicación. Un estudio en los informativos de TVE Pixel-bit. Revista de Medios y Educación, 34, 121-134. http://www.redalyc.org/articulo. oa? id=36812036009

MacBride, S. (1980). Un solo mundo, voces múltiples. Comunicación e información en nuestro tiempo. Ciudad de México: Fondo de Cultura Económica.

Martínez, L. (2005). La participación de los usuarios en los contenidos periodísticos de la red. En López García, G. (ed.) El ecosistema digital: modelos de comunicación, nuevos medios y público en Internet. Valencia: Servei de Publicacions de la Universitat de Valencia. Recuperado de http://www.uv.es/de- mopode/libro1/LourdesMartinez.pdf

Mendiguren, T., Pérez, J. \& Peña, S. (2011). Renovarse o morir: la transformación de la prensa tradicional con presencia en Internet hacia un modelo más participativo. En Verón, J. Y Sabés, F. (coord), La investigación en Periodismo Digital. Asociación de la Prensa de Aragón, España, pp. 421-431.

Molina, C. (1994). Dialéctica feminista de la ilustración. Madrid: Antrophos.

Morahan-Martin, J. (2000). Women and the internet: Promise and perils. Cyber Psychology \& Behavior, 3 (5), 683-691.

UN. CEPAL (2013). Mujeres en la economía digital: superar el umbral de la desigualdad. 158 p. LC/L.3666(CRM.12/3). Recuperado de https://repositorio.cepal.org/handle/11362/16561

Otner, S. (1979) ¿Es la mujer con respecto al hombre lo que la naturaleza con respecto a la cultura?. En Harris, Olivia y Kate Young (Comps.). Barcelona: Anagrama, pp. 109-131.

Pateman, C. (1996). Críticas feministas a la dicotomía público/privado. En Pateman, C. (coord). Críticas feministas a la dicotomía público/privado. Barcelona: Paidos, pp. 2-23. 
Pierson, E (2015). Outnumbered But Well-Spoken : Female Commenters in the New York Times. In Proc. Computer Supported Cooperative Work and Social Computing (CSCW).

Raimondo, N. (2012). La prensa online y su público. Un estudio de los espacios de intervención y participación del lector en los diarios argentinos Clarín y La Nación. Buenos Aires: Teseo.

Raimondo, N. (2014). Prensa online y tipos de lectores. Respuestas del público y lógicas de reconocimiento en los comentarios a las noticias del diario argentino La Nación. Cuadernos.info, 34, 183-195. doi: 10.7764/cdi.34.535

Red de Investigadoras por la Vida y la Libertad de las Mujeres. (2008). Por los derechos humanos de las mujeres y las niñas: su acceso a la comunicación y la información. Lineamientos y mecanismos para una legislación de medios con perspectiva de género. México. Recuperado de http://americalatinagenera.org/newsite/images/leydemediosconperspectivadegenero.pdf

Scott, J. (1990). El género: Una categoría útil para el análisis histórico. En Lamas, M. Compiladora. El género: la construcción cultural de la diferencia sexual. PUEG, México. pp. 265-302.

Selfe, C. \& Meyer, P. (1991).Testing claims for online conferences. Written Communication 8 (2), pp. 163-192.

Sreberny-Mohammadi, A. (1996). Comunicación y desarrollo un vínculo necesario. En Portugal, A y Torres, C. (eds.) Por todos los medios: comunicación y género. Edición de las Mujere: Isis Internacional, pp.17-35

Vega, A. (2010). Las mujeres y el derecho humano a la comunicación: su acceso y participación en la industria mediática. Revista Mexicana de Ciencias Políticas y Sociales, (52) 208, pp. 81-95 Universidad Nacional Autónoma de México, Distrito Federal, México.

Yates, S.J. (1997). Gender, Indentity and CMC. Journal of Computer Assisted Learning (13) 281-290.

- Sobre la autora:

Karen Gheza Correa es publicista, Licenciada en Comunicación Persuasiva de la Universidad del Pacífico, Magíster en Comunicación Social de la Universidad de Chile. Experiencia docente en Marketing Digital [USACH]. Ponente en el IV Congreso Internacional de Estudios sobre Medios de Comunicación, 2019, Bonn, Alemania.

- ¿Como citar?

Gheza Correa, K. (2019). Diferencias de género en las prácticas e interacciones en los comentarios del diario online Emol.com. Comunicación y Medios, (40), 140-154. 